\title{
Zernike polynomials for photometric characterization of LEDs
}

\author{
J.L. Velázquez, A. Ferrero, A. Pons, J. Campos and M.L. \\ Hernanz \\ Departamento de Imágenes, Visión e Instrumentación Óptica, Instituto de Óptica \\ (CSIC), c/ Serrano 144, Madrid, 28006, Spain \\ E-mail: jl.velazquez@csic.es
}

\begin{abstract}
We propose a method based on Zernike polynomials to characterize photometric quantities and descriptors of LEDs from measurements of the angular distribution of the luminous intensity, such as total luminous flux, beam angle, inhomogeneity, anisotropy, direction of the optical axis and Lambertianity of the source. The performance of this method was experimentally tested for eighteen high-power LEDs from different manufacturers and with different photometric characteristics. A small set of Zernike coefficients can be used to calculate all the mentioned photometric quantities and descriptors. For applications not requiring a great accuracy such as those of lighting design, the angular distribution of the luminous intensity of most of the studied LEDs can be interpolated with only two Zernike polynomials.
\end{abstract}




\section{Introduction}

Recent advances in Light Emitting Diodes (LEDs) technology have spread their use in a growing number of applications. This kind of light source presents some important advantages with respect to conventional ones, as better luminous efficiency, longer lifetime, lower cost and smaller size, which allows these devices to be easily adapted in different application areas, as lighting, automotive industry, aeronautics, road safety, health, fashion, etc. However, the emission of LEDs differs from the other previous conventional sources, and the implantation on those illumination systems requires the re-definition of standard photometric procedures to accurately characterize them $[1,2,3,4,5,6]$. Because of that, the International Commission on Illumination (CIE) led a process to establish a recommendation on the procedure of measurement of LED's emission in 2007 [7], establishing preliminary standard procedures for laboratories and manufacturers to photometrically characterize them. Later on, CIE has promoted the publication of a CIE International Standard [8] to provide requirements to perform reproducible photometric and colorimetric measurements on LED lamps, LED modules, and LED luminaries, since the availability of reliable and accurate photometric data for LED devices is a basic requirement for designing good lighting systems and evaluating performance of products.

Under a photometric point of view the determination of the illuminance produced by the source at a given position is one of the key points in most of the previous applications. This can be done if the angular distribution of the luminous intensity is known. At present this distribution has to be measured by using gonio-photometers since LED devices are diverse and emission models are not available for every one, so a dense knowledge of the distribution has to be learnt from measurements.

Transformation of luminous intensity distribution from spherical coordinates into polar coordinates is proposed here, so that photometric features of LEDs can be easily perceived as deviations from rather standard patterns. This situation resembles the problem of studying aberrations in optical systems, where the deviations of a real image respect to a standard pattern are classified.

Zernike polynomials [9] are a complete set of orthogonal polynomials over the interior of a unit circle, widely used in the study and specification of aberrations [10] and other physical problems [11]. The luminous intensity distribution also can be represented over a circle of unity radius and, in addition, Zernike polynomials are interesting in principle for specifying photometric features of LEDs due to their analytical properties (certain simple properties of invariance) derived from the simplicity of the radial functions and the factorization in radial and azimuthal functions. As we will show in this article, this allows beam angle, optical axis direction, inhomogeneity, anisotropy or Lambertianity degree of LEDs to be expressed by using a low number of polynomials. And more importantly, Zernike polynomials provided a well-established basis to interpolate luminous intensity LEDs with low number of terms. Other orthogonal bases such as Bessel functions, for instance, could be checked, but less 
compact expressions for photometric features would be expected because of their definition.

To test the feasibility of this method, the luminous intensity distribution of eighteen high-power LEDs from different manufacturers (Cree Xlamp®), Philips Lumileds LUXEON Rebel@, Osram Golden Dragon $($ ) ) and with different photometric properties have been measured with a gonio-photometer developed at our laboratory [12]. Then Zernike polynomials were fitted to luminous intensity distribution measurements and photometric features and quantities were calculated. The results so obtained are shown in section 3 of this work and compared to reference values where possible.

\section{Zernike polynomials for describing optical properties of LEDs}

Zernike polynomials can be expressed as separable functions of polar coordinates as [13]:

$$
Z_{n}^{l}(\rho, \vartheta)=R_{n}^{l}(\rho) \exp (i l \vartheta)
$$

where $\rho$ is the radial coordinate, $\vartheta$ is the angular coordinate, $n$ is a positive integer, and $l$ can be a positive or negative integer whose $|l|$ yields an even value of $n-|l| . R_{n}^{l}(\rho)$ is defined by $(m=|l|)$ :

$$
R_{n}^{ \pm m}(\rho)=\sum_{k=0}^{\frac{n-m}{2}} \frac{(-1)^{k}(n-k) !}{k !\left(\frac{n+m}{2}-k\right) !\left(\frac{n-m}{2}-k\right) !} \rho^{n-2 k}
$$

For the interested reader, some properties of the Zernike polynomials are summarized in [13]. But the most interesting property to our aims is that any sufficiently smooth real-valued field $F(\rho, \vartheta)$ over the unit disk can be represented in terms of its Zernike coefficients $C_{\mathrm{n}, \mathrm{m}}$ as:

$$
F(\rho, \vartheta)=\sum_{n, m} C_{\mathrm{n}, \mathrm{m}} Z_{\mathrm{n}}^{\mathrm{m}}(\rho, \vartheta)
$$

The luminous intensity distribution $I_{V}(\theta, \varphi)$ is given in spherical coordinates (polar angle $\theta$ and azimuth angle $\varphi$ ). However, since there is a single value of $I_{V}$ for every direction, $I_{V}(\theta, \varphi)$ can be expressed in polar coordinates (radius $\rho$ and polar angle $\vartheta$ ) as:

$$
I_{V}(\theta, \varphi)=I_{V}(\rho, \vartheta)
$$

by using the transformation:

$$
\begin{aligned}
& \rho=\sin \theta \\
& \vartheta=\varphi
\end{aligned}
$$

Then, the distribution is defined in a circle of unit radius and according to Eq. 3 can be expressed as:

$$
I_{V}(\theta, \varphi)=I_{V}(\sin \theta, \varphi)=\sum_{n, m} C_{\mathrm{n}, \mathrm{m}} Z_{\mathrm{n}}^{\mathrm{m}}(\sin \theta, \varphi)
$$

The advantage of expressing $I_{V}(\theta, \varphi)$ in this way is that the complexity of the angular distribution is characterized by only some scalars, the Zernike coefficients $C_{\mathrm{n}, \mathrm{m}}$, 
whose interpretation regarding their partial contributions to the wavefront is wellknown [9]. Therefore, the key is to have a low number of polynomials involved in the characterization of the distribution, which in turn will depend on the distribution itself and on the accuracy required in the application. In this article, we will express photometric features and quantities as a function of only nine Zernike coefficients, because increasing the number of polynomials does not yield a better fitting to the luminous intensity distribution. The polynomials related with those coefficients are given in Table 1. We must notice that, for most of the 18 studied LEDs, the angular distribution of the luminous intensity can be interpolated with just two Zernike polynomials $\left(P_{2}\right.$ and $P_{4}$, in Table 1 ), as it will be shown in section 3 .

From the subset in Table 1, the relevant photometric characteristics and quantities can be estimated as shown below.

\subsection{Beam angle}

We will determine the beam angle (BA) as the full angular width between values of the luminous intensity whose magnitude is half the distribution maximum, which is usually written as FWHM. This is the definition adopted in CIE standard on LED test methods [8]. Taking into account that LEDs generally have got rotational symmetry and divergence is defined just on the polar coordinate, only polynomials $\left(P_{0}, P_{4}\right.$ and $\left.P_{8}\right)$ are relevant to calculate this characteristics (see Table 1).

$$
P_{0}+P_{4}\left(\sin \theta_{\mathrm{BA}}\right)+P_{8}\left(\sin \theta_{\mathrm{BA}}\right)=\frac{\max \left[I_{V}(\theta, \varphi)\right]}{2}
$$

From this equation, the analytical solution:

$$
\theta_{\mathrm{BA}}=\arcsin \sqrt{\varepsilon\left(1 \pm \operatorname{sgn}(\varepsilon) \sqrt{1+\frac{\gamma}{\varepsilon^{2}}}\right)}
$$

is obtained, where the variables $\varepsilon$ and $\gamma$ are defined in terms of Zernike coefficients as:

$$
\begin{aligned}
& \varepsilon=\frac{1}{2}-\frac{1}{2 \sqrt{15}} \frac{C_{2,0}}{C_{4,0}} \\
& \gamma=\frac{-2 C_{0,0}+\sqrt{12} C_{2,0}-\sqrt{20} C_{4,0}+\max \left[I_{V}(\theta, \varphi)\right]}{12 \sqrt{5} C_{4,0}}
\end{aligned}
$$

If the luminous intensity distribution is symmetrical and the mechanical axis coincides with the optical axis, the expression of the beam angle is obtained as:

$$
B A=\theta_{B A+}-\theta_{B A-}
$$

\subsection{Direction of the optical axis}

The optical axis aims to show the main direction along which the luminous source emits and it does not always exactly coincide with the mechanical axis referred to the source socket. Optical axis depends on the angular radiation pattern of LEDs and has got real sense only for those LEDs emitting in a single lobe. Not always the emission in a 
single lobe is smooth, so in this work the optical axis will be defined as the direction corresponding to the weighted mean defined by the following equations:

$$
\begin{aligned}
& x_{c}=\frac{\int x I_{\mathrm{V}}(x, y) \mathrm{d} A}{\int I_{\mathrm{V}}(x, y) \mathrm{d} A}=\frac{\int_{0}^{2 \pi} \int_{0}^{1} \rho \cos \vartheta I_{V}(\rho, \vartheta) \rho \mathrm{d} \rho \mathrm{d} \vartheta}{\int_{0}^{2 \pi} \int_{0}^{1} I_{V}(\rho, \vartheta) \rho \mathrm{d} \rho \mathrm{d} \vartheta}=\frac{C_{1,1}}{2 C_{0,0}} \\
& y_{c}=\frac{\int y I_{\mathrm{V}}(x, y) \mathrm{d} A}{\int I_{\mathrm{V}}(x, y) \mathrm{d} A}=\frac{\int_{0}^{2 \pi} \int_{0}^{1} \rho \sin \vartheta I_{V}(\rho, \vartheta) \rho \mathrm{d} \rho \mathrm{d} \vartheta}{\int_{0}^{2 \pi} \int_{0}^{1} I_{V}(\rho, \vartheta) \rho \mathrm{d} \rho \mathrm{d} \vartheta}=\frac{C_{1,-1}}{2 C_{0,0}}
\end{aligned}
$$

which are the cartesian coordinates of the luminous intensity centroid. The point is related with the direction of the axis as:

$$
\theta_{0}=\arcsin \left(\sqrt{x_{\mathrm{c}}^{2}+y_{\mathrm{c}}^{2}}\right)=\arcsin \left(\frac{\sqrt{C_{1,1}^{2}+C_{1,-1}^{2}}}{2 C_{0,0}}\right)
$$

and

$$
\varphi_{0}=\arctan \left(\frac{y_{\mathrm{c}}}{x_{\mathrm{c}}}\right)=\arctan \left(\frac{C_{1,-1}}{C_{1,1}}\right)
$$

We must notice that, for this last equation, the sign of $x_{c}$ and $y_{c}$ has to be taken into account to assign the value to azimuthal angle $\left(0 \leq \varphi_{0}<2 \pi\right)$. Therefore, the optical axis direction is given just by three Zernike coefficients: $C_{0,0}, C_{1,1}, C_{1,-1}$.

\subsection{Inhomogeneity}

In some applications it may be interesting to have an idea about the closeness of LED sources to an ideal point source whose luminous intensity would be constant at every direction. To quantify this we will define this inhomogeneity term, $\mathrm{U}$, as the square root of the summation of all Zernike weighted polynomials except the term $C_{0,0}$, which represents the mean value.

$$
U=\sqrt{\sum_{\mathrm{i}=1}^{\mathrm{i}=8} \frac{\int_{0}^{1} \int_{0}^{2 \pi} P_{\mathrm{i}}^{2}(\rho, \vartheta) \rho \mathrm{d} \rho \mathrm{d} \vartheta}{\int_{0}^{1} \int_{0}^{2 \pi} P_{0}^{2}(\rho, \vartheta) \rho \mathrm{d} \rho \mathrm{d} \vartheta}}
$$

Applying orthogonality property of Zernike polynomials the following expression is obtained:

$$
U=\sqrt{\sum_{\mathrm{n} \neq 0} \sum_{\mathrm{m}} \hat{C}_{\mathrm{n}, \mathrm{m}}^{2}}
$$

where $\hat{C}_{\mathrm{n}, \mathrm{m}}=\frac{C_{\mathrm{n}, \mathrm{m}}}{C_{0,0}}$. This yields a value of $U \approx 0$ when the emission comes to a spherical wavefront.

\subsection{Anisotropy}

An important characteristic of the luminous intensity distribution is to know whether there is rotational symmetry. We will define the anisotropy coefficient as the relative variation of the luminous intensity with respect the azimuthal angle $(\varphi)$, and, since it may depend on $\theta$, one value should be calculated for every $\theta$. The coefficient will 
be expressed by the contribution of those Zernike polynomials which depend on the azimuth angle, which are all except for $P_{0}, P_{4}$ and $P_{8}$ :

$$
\begin{aligned}
& A(\rho)=\sqrt{\sum_{\mathrm{i} \neq 0,4,8} \frac{\int_{0}^{2 \pi} P_{i}^{2}(\rho, \vartheta) \rho \mathrm{d} \vartheta}{\left[P_{0}^{2}(\rho)+P_{4}^{2}(\rho)+P_{8}^{2}(\rho)\right]}} \\
& =\sqrt{\frac{2 \pi \rho^{3}\left(8 \rho_{0}^{2}+\alpha_{\mathrm{c}} \hat{C}_{c}^{2}+\alpha_{\mathrm{a}} \hat{C}_{a}^{2}\right)}{1+\alpha_{\mathrm{d}} \hat{C}_{2,0}^{2}+\alpha_{\mathrm{s}} \hat{C}_{4,0}^{2}}}
\end{aligned}
$$

where:

$$
\begin{aligned}
& \rho_{0}=\sin \theta_{0} \quad(\text { see Eq. 15) } \\
& \hat{C}_{\mathrm{c}}^{2}=\hat{C}_{3,1}^{2}+\hat{C}_{3,-1}^{2} \\
& \hat{C}_{\mathrm{a}}^{2}=\hat{C}_{2,-2}^{2}+\hat{C}_{2,2}^{2} \\
& \alpha_{\mathrm{c}}=4\left(2-3 \rho^{2}\right)^{2} \\
& \alpha_{\mathrm{a}}=3 \rho^{2} \\
& \alpha_{\mathrm{d}}=3\left(-1+2 \rho^{2}\right)^{2} \\
& \alpha_{\mathrm{s}}=5\left(1-6 \rho^{2}+6 \rho^{4}\right)^{2}
\end{aligned}
$$

\subsection{Total luminous flux}

The total luminous flux $\Phi_{V}$ of the $L E D$ can be calculated by integration of Eq. (7) with respect to the projected solid angle $\mathrm{d} \Omega=\sin \theta \mathrm{d} \theta \mathrm{d} \varphi=\rho \mathrm{d} \rho \mathrm{d} \vartheta$. The $\varphi$-dependent terms are cancelled out in this integration and the total luminous flux is given by the very simple equation:

$$
\Phi_{V}=\int_{0}^{2 \pi} \mathrm{d} \vartheta \int_{0}^{1} I_{V}(\rho, \vartheta) \rho \mathrm{d} \rho=2 \pi\left(C_{0,0}+\frac{1}{\sqrt{3}} C_{2,0}+\frac{1}{\sqrt{5}} C_{4,0}\right)
$$

\subsection{Lambertianity degree}

According to the Lambert's cosine law [14], the luminous intensity of an ideal radiator is directly proportional to the cosine of $\theta$. A common procedure to quantify the Lambertianity degree of a source is to calculate the parameter $g$ in the equation:

$$
I_{V}(\theta)=I_{V 0} \cos ^{g} \theta
$$

The resolution of this equation for the parameter $g$ can be expressed as a function of beam angle (and, in consequence, of Zernike coefficients, as it was given in subsection 2.1) in the following way:

$$
\frac{I_{\mathrm{V} 0}}{2}=I_{\mathrm{V} 0} \cos ^{g}\left(\frac{B A}{2}\right)
$$




$$
g=\frac{-\ln 2}{\ln \left(\cos \frac{B A}{2}\right)}
$$

\section{Results}

To check the validity of the Zernike approach, luminous intensity distribution of a set of LEDs has been measured in the goniophotometer developed at CSIC [12]. This instrument is composed of a temperature-stabilized photometer and two stepper motor-based rotation stages, which allow the illuminance at any emission direction to be measured except for those occluded by the source. The measurement quality of the goniophotometer was validated by comparing with PTB (Physikalisch-Technische Bundesanstalt) measurements of luminous intensity distributions of four stable LEDs, as it was reported in [12]. Illuminance measurements by varying the polar angle between $0^{\circ}$ and $90^{\circ}$ with a $5^{\circ}$ step and the azimuth angle between $-180^{\circ}$ and $180^{\circ}$ with a $12^{\circ}$ step were taken at a distance of $1.9 \mathrm{~m}$ for 18 LEDs from different manufacturers, whose instability was characterized as less than $0.1 \%$. As an example, the luminous intensity angular distributions $(C-\gamma$ photometric data) of three LEDs from different manufacturers are shown in Figures 1.a), 1.c) and 1.e), where, in the representation, the radial position $(\rho)$ corresponds with $\sin \theta$.

Luminous intensity distributions have been transformed according to Eq. 4 and Zernike polynomials have been fitted to them. The relative differences of this fitting for the same three LEDs are shown in 2-D plots in Figures 1.b), 1.d) and 1.f), respectively. Fitting is very good for the Philips Luxeon Rebel LED, which is the one having the softest variation on luminous intensity distribution. Fitting is also very good for the Osram Golden Dragon LED till $80^{\circ}$ polar angle, where a sharp change in luminous intensity is produced. The same behaviour is found for Cree Xlamp LED: the fitting is good as far as the luminous intensity varies smoothly.

To give a clearer idea of the differences between experimental values of luminous intensity and the ones obtained from Zernike fitting, they are plotted versus the polar angle in Fig. 3. Error bars represent the standard deviations over the azimuthal angle. As mentioned before, the worst case is observed in the LED from Osram where a sudden drop of the luminous intensity occurs at $\theta=75^{\circ}$.

The goodness of the fitting obtained by the Zernike approach is shown in Figure 2. The relative sum of squared errors (SSE) (in semi-logarithm scale) is given for the 18 studied LEDs as a function of the number of Zernike terms used in the fitting. The relative SSE with only one term corresponds to the constant term $\left(C_{0,0}\right)$; with two terms, we added the term corresponding to the coefficient $C_{2,0}$, and so on until all the significant Zernike terms are used. The order in which terms are added is specified at the secondary $\mathrm{X}$-axis on the top of the plot. This order was determined by the average value of the Zernike coefficients of the 18 studied LEDs, sorted from larger to smaller values. 
According to Fig. 2, the coefficients $C_{0,0}$ and $C_{2,0}$ are the most significant for most of the LEDs studied, being negligible the improvement of the fitting by increasing the number of polynomials in the case of Osram LEDs. In addition, for Osram and Luxeon LEDs, we obtained that the relative SSE lies always below $4 \%$ when we used just the five first Zernike terms. For all of these LEDs but one (warm white Luxeon), the number of polynomials to fit the angular distribution of the luminous intensity can be reduced to just $2\left(C_{2,0}\right.$ and $\left.C_{4,0}\right)$ if a relative SSE of $5 \%$ is allowed. For Cree LEDs, however, the result is different. The relative SSE of the fitting is not significantly improved by increasing the number of polynomials involved, reaching a value over $7 \%$ in any case. The fitting is not appreciably improved for any of the studied LEDs when using more than nine terms.

\subsection{Beam angle}

Beam angle values obtained from direct measurement and Zernike-based analysis for the measured LEDs are given in Table 2. These LEDs have got beam angle values ranging between $70^{\circ}$ and $150^{\circ}$ as can be seen for some of them in Figure 3, as well as their difference. The best agreement is obtained for beam angle of LEDs from Philips, with an average error of $4^{\circ}$, which is consistent with the angular resolution of the measurement $\left(5^{\circ}\right)$. For most of the Osram LEDs, Eq. 9 is undetermined because the values of the intensity are always above half maximum for Zernike data and $B A$ can not be calculated. Average deviation in LEDs from Cree is $-9^{\circ}$, with individual deviations from $-2.5^{\circ}$ to $-13.5^{\circ}$.

\subsection{Optical axis}

Optical axis directions of the measured LEDs, calculated by Eqs. 15 and 16, are randomly distributed around the mechanical axis (origin of coordinates), as shown in the polar plot of Fig. 4. The higher angular deviation between both axis is around $2.5^{\circ}$. Most of the LEDs from Philips present an angular deviation between $0^{\circ}$ and $1^{\circ}$. Angular deviations of LEDs from Osram are more or less uniformly distributed between $0^{\circ}$ and $0.5^{\circ}$.

\subsection{Inhomogeneity}

The relation between inhomogeneity data, calculated on one hand by Eq. $18\left(U_{\text {Zer }}\right)$ and on other hand as the relative standard deviation of the measured luminous intensity $\left(U_{\text {std }}\right)$, is shown in Fig. 5 for one representative LED of each manufacturer. There is a proportionality between them, but with a constant lower than 1 for all LEDs. This may be partially explained by the distribution of the measurement directions over which $U_{\text {std }}$ is calculated. Notice that, although the spherical coordinates are uniformly distributed, those directions are not, but have higher density for lower polar angles. As expected, the smaller the beam angle, the more inhomogeneous is the emission. 


\subsection{Anisotropy}

Anisotropy data, calculated both by Eq. 19 ( $A_{\text {Zer }}$, as solid lines) and as the relative standard deviation of the luminous intensity over different azimuth angles $\left(A_{\text {std }}\right.$, as crosses, triangles and circles), are plotted versus polar angle in Figure 6 for a representative LED of each manufacturer. For every LED, it lies below $2 \%$ for polar angles lower than $50^{\circ}$, and it increases faster towards larger angles. The Zernikebased calculations are close to the measurement based ones for polar angles up to $75^{\circ}$ approximately. This is consistent with the results shown in Figure 1 (b, d and e) that shows a larger deviation of the fitting at higher polar angles.

\subsection{Total luminous flux}

Total luminous flux values, calculated both by integration of the angular distribution of the luminous intensity and by the proposed Zernike-based procedure (Eq. 20) are displayed in Table 3. Zernike coefficients allow the luminous flux of a LED to be estimated with an error lower than $4 \%$ for Philips's LEDs. For Cree's LEDs the difference is higher and with opposite sign. Important deviations are found for Osram's LEDs (average error of $14 \%$ ), due to the mentioned sudden drop of the luminous intensity at high polar angles, that the Zernike procedure is unable to represent.

\subsection{Lambertianity}

Parameter $g$ for the studied LEDs calculated by both a least squares fitting to the experimental luminous intensity distribution and by the Zernike-based $\left(g_{\text {Zer }}\right)$ procedure (Eq. 23) are shown in Table 4. For Philips LEDs, which are almost Lambertian because their $g$ values are closer to 1 , the difference between the parameter $g$ calculated by both methods is lower than the uncertainty of the parameter calculated by LSF, so they can be considered equivalent. For Cree LEDs, whose parameter $g$ is higher than 1 , the difference between both $g$ values is higher than the fitting uncertainty for $g$ values higher than 2. For Osram LEDs, $g$ value cannot be calculated with Eq. 23 because $B A$ could not be determined.

\section{Applications}

Calculated Zernike coefficients specify the luminous intensity distribution of a LED. We think that the composed function of Zernike polynomials may be an alternative to the commonly used procedure to render illumination at large surfaces. The common approach is to use a ray file (list of rays characterized by a direction of propagation and a luminous flux) in a simulation software to calculate by ray-tracing the illuminance at given positions. However, this technique is very time-consuming and, consequently, slows for real-time visualization programs. Using the Zernike function of a source, it is possible to calculate by integration the illuminance anywhere. It would reduce 
considerably the rendering time. A faster rendering provides a great advance in the lighting design, allowing improvement in the illumination efficiency and in the achieved illumination uniformity.

\section{Conclusions}

We have proposed a method based on Zernike polynomials to characterize photometric quantities and descriptors of LEDs, such as total luminous flux, beam angle, anisotropy, direction of the optical axis and lambertianity degree of the source.

The performance of this method has been experimentally tested using luminous intensity measurements of eighteen high-power LEDs from different manufacturers and with different optical characteristics, carried out in a goniophotometer developed at IO-CSIC.

We have found that a small set of nine Zernike coefficients can be enough to calculate all the mentioned photometric quantities and descriptors. In addition, we have shown that, when the application does not required low uncertainty values, a reasonable approach to the angular distribution of the luminous intensity can be obtained with just two Zernike polynomials for most of the eighteen studied LEDs, being necessary between 5 and 9 polynomials to get a fitting error that cannot be decreased by increasing the number of polynomials used.

We have to notice that some shortcomings are found in the method, for instance, when the luminous intensity has a 'hat' distribution, as we found in the case of the Osram LEDs. Despite these shortcomings, we think that this Zernike-based approach may be an alternative to the probably more time-consuming ray-tracing procedures to render illumination at large surfaces.

\section{Acknowledgements}

This work was accomplished within the EMRP ENG05 Project "Metrology for Solid State Lighting". The EMRP is jointly funded by the EMRP participating countries within EURAMET and the European Union. The authors are also grateful to Comunidad de Madrid for funding the project SINFOTON-CM: S2013/MIT-2790.

\section{References}

[1] D R Agaphonov, V S Ivanov, V I Sapritsky, and R I Stolyarevskaya. Light measurements of high-luminance LEDs . Metrologia, 37(5):587, 2000.

[2] Pasi Manninen, Jari Hovila, Lauri Seppälä, Petri Kärhä, Lasse Ylianttila, and Erkki Ikonen. Determination of distance offsets of diffusers for accurate radiometric measurements. Metrologia, 43(2):S120, 2006.

[3] P Manninen, J Hovila, P Kärhä, and E Ikonen. Method for analysing luminous intensity of light-emitting diodes. Measurement Science and Technology, 18(1):223, 2007.

[4] Yoshi Ohno. Optical metrology for LEDs and solid state lighting. Proceedings SPIE, 6046:604625-604625-8, 2006. 
[5] K. Godo, T. Saito, H. Shitomi, T. Zama, and I. Saito. Development of a total luminous flux measurement facility for LEDs at the national metrology institute of japan. Proceedings of the 12th International Conference on New Developments and Applications in Optical Radiometry (NEWRAD 2014), 1:95, 2014.

[6] Miran Bürmen, Franjo Pernuš, and B. Likar. Automated optical quality inspection of light emitting diodes. Measurement Science and Technology, 17(6):1372, 2006.

[7] Publication CIE 127:2007. Measurement of LEDs . Technical report, Vienna: CIE, 2007.

[8] Publication CIE S 025/E:2015. Test method for LED lamps, LED luminaires and LED modules. Technical report, Vienna: CIE, 2015.

[9] C-J Kim and R. R. Shannon. Catalog of Zernike polynomials. Applied Optics and Optical Engineering, X:193-221, 1987.

[10] Virendra N. Mahajan. Zernike annular polynomials for imaging systems with annular pupils. J. Opt. Soc. Am., 71(1):75-85, Jan 1981.

[11] Robert J. Noll. Zernike polynomials and atmospheric turbulence. J. Opt. Soc. Am., 66(3):207-211, Mar 1976.

[12] J. L. Velázquez, A. Ferrero, M. López, A. Pons, and A. et al. Villamarín. Angular distribution of the averaged luminous intensity of low power LEDs transfer standards. Proc. SPIE 8785, 8th Iberoamerican Optics Meeting and 11th Latin American Meeting on Optics, Lasers, and Applications, 8785:87858W, November 18, 2013.

[13] Max Born and Emil Wolf. Principles of optics: electromagnetic theory of propagation, interference and diffraction of light. CUP Archive, 1999.

[14] James M Palmer and Barbara Geri Grant. The art of radiometry. SPIE Press Bellingham, 2010 . 
Table 1. Nomenclature for the Zernike polynomials used in this article.

\begin{tabular}{ll}
\hline Term of Zernike & Contribution to distribution \\
\hline$Z_{0}^{0}=1$ & $P_{0}=C_{0,0} Z_{0}^{0}$ \\
$Z_{1}^{-1}(\sin \theta, \varphi)=2 \sin \theta \sin \varphi$ & $P_{1}(\rho, \vartheta)=P_{1}(\sin \theta, \varphi)=C_{1,-1} Z_{1}^{-1}(\sin \theta, \varphi)$ \\
$Z_{1}^{1}(\sin \theta, \varphi)=2 \sin \theta \cos \varphi$ & $P_{2}(\rho, \vartheta)=P_{2}(\sin \theta, \varphi)=C_{1,1} Z_{1}^{1}(\sin \theta, \varphi)$ \\
$Z_{2}^{-2}(\sin \theta, \varphi)=\sqrt{6} \sin ^{2} \theta \sin 2 \varphi$ & $P_{3}(\rho, \vartheta)=P_{3}(\sin \theta, \varphi)=C_{2,-2} Z_{2}^{-2}(\sin \theta, \varphi)$ \\
$Z_{2}^{0}(\sin \theta)=\sqrt{3}\left(2 \sin ^{2} \theta-1\right)$ & $P_{4}(\rho)=P_{4}(\sin \theta)=C_{2,0} Z_{2}^{0}(\sin \theta)$ \\
$Z_{2}^{2}(\sin \theta, \varphi)=\sqrt{6} \sin ^{2} \theta \cos 2 \varphi$ & $P_{5}(\rho, \vartheta)=P_{5}(\sin \theta, \varphi)=C_{2,2} Z_{2}^{2}(\sin \theta, \varphi)$ \\
$Z_{3}^{-1}(\sin \theta, \varphi)=\sqrt{8}\left(3 \sin ^{3} \theta-2 \sin \theta\right) \sin \varphi$ & $P_{6}(\rho, \vartheta)=P_{6}(\sin \theta, \varphi)=C_{3,-1} Z_{3}^{-1}(\sin \theta, \varphi)$ \\
$Z_{3}^{1}(\sin \theta, \varphi)=\sqrt{8}\left(3 \sin ^{3} \theta-2 \sin \theta\right) \cos \varphi$ & $P_{7}(\rho, \vartheta)=P_{7}(\sin \theta, \varphi)=C_{3,1} Z_{3}^{1}(\sin \theta, \varphi)$ \\
$Z_{4}^{0}(\sin \theta)=\sqrt{5}\left(6 \sin ^{4} \theta-6 \sin ^{2} \theta+1\right)$ & $P_{8}(\rho)=P_{8}(\sin \theta)=C_{4,0} Z_{4}^{0}(\sin \theta)$ \\
\hline
\end{tabular}

Table 2. Comparison between the beam angle obtained by direct measurement and Zernike-based analysis.

\begin{tabular}{|c|c|c|c|c|}
\hline & & Beam ang & $e / 0$ & \\
\hline & & Direct & Zernike & Absolute \\
\hline & Polar White & 93.9 & 80.4 & -13.5 \\
\hline & White & 92.3 & 81.8 & -10.5 \\
\hline & Warm White & 89.6 & 76.8 & -12.8 \\
\hline Cree Xlamp & Neutral White & 85.2 & 75.6 & -9.6 \\
\hline & Blue & 93.5 & 83.0 & -10.5 \\
\hline & Red & 75.9 & 73.4 & -2.5 \\
\hline & Green & 96.1 & 92.4 & -3.7 \\
\hline & Polar White & 101.3 & 105.7 & 4.4 \\
\hline & Warm White & 114.0 & 118.1 & 4.1 \\
\hline Philins I uyeon Robol & Neutral White & 106.6 & 111.8 & 5.2 \\
\hline FImps Luxeon ReDel & Blue & 112.3 & 116.5 & 4.2 \\
\hline & Red & 118.4 & 121.3 & 2.9 \\
\hline & Green & 110.6 & 113.5 & 2.9 \\
\hline & White & 150.7 & - & - \\
\hline & Warm White & 145.5 & 157.3 & 11.8 \\
\hline Osram Golden Dracon & Blue & 152.2 & - & - \\
\hline Usram Golden Dragon & Red & 151.8 & - & - \\
\hline & Green & 147.0 & - & - \\
\hline
\end{tabular}


Table 3. Total flux comparison between experimental and the Zernike-based values

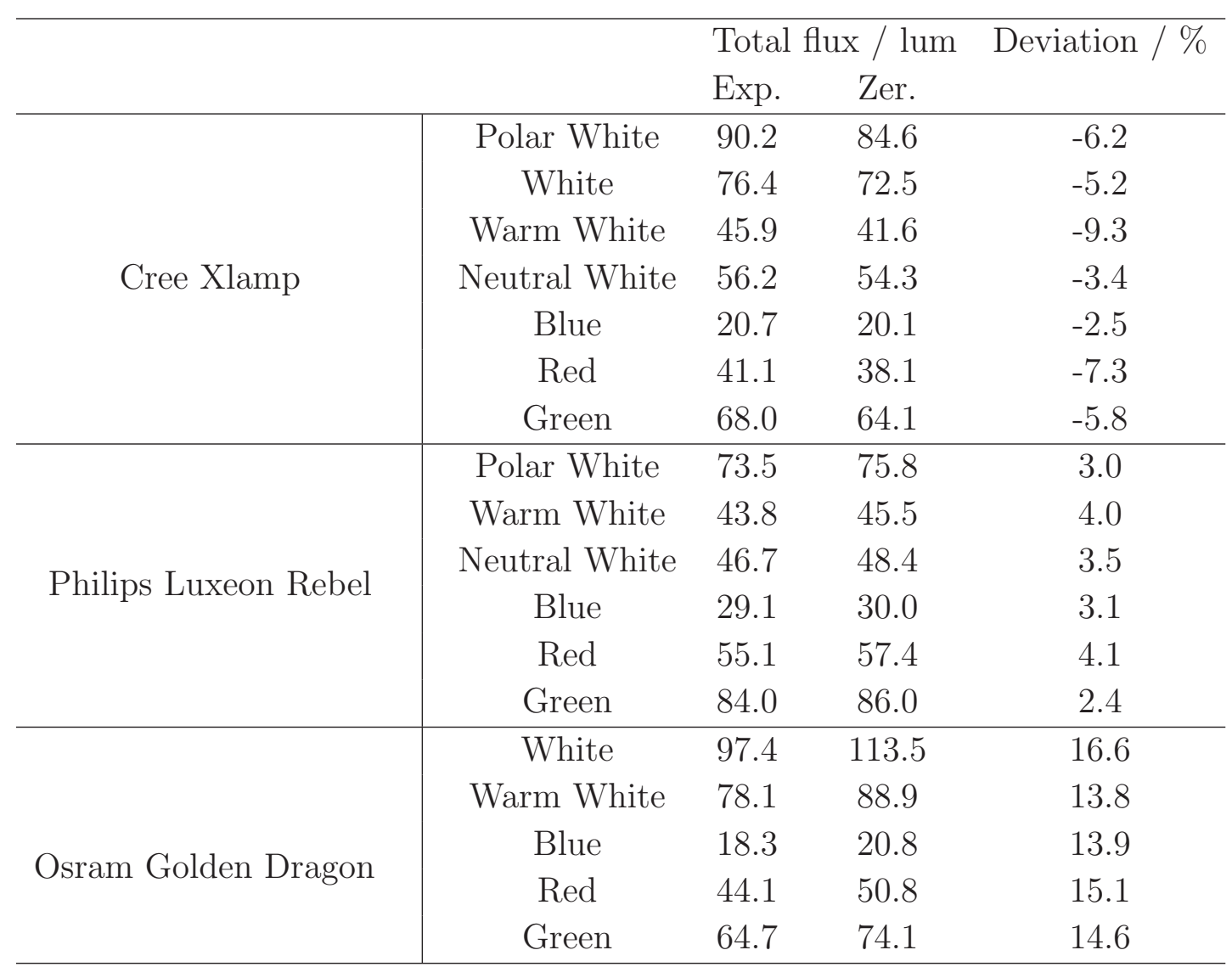


Table 4. Lambertianity of the luminous intensity distribution of all studied LEDs, where $g$ is the exponent in the cosine law.

\begin{tabular}{c|cccc}
\hline \multicolumn{1}{c}{} & & $g_{\text {LSF }} \pm U(\mathrm{k}=2)$ & $g_{\text {Zer }}$ & $\begin{array}{c}\text { Absolute } \\
\text { deviation }\end{array}$ \\
\hline \multirow{5}{*}{ Cree Xlamp } & Polar White & $2.22 \pm 0.30$ & 2.6 & 0.38 \\
& White & $2.14 \pm 0.29$ & 2.47 & 0.33 \\
& Warm White & $2.40 \pm 0.24$ & 2.85 & 0.45 \\
& Neutral White & $2.49 \pm 0.32$ & 2.94 & 0.45 \\
& Blue & $2.03 \pm 0.29$ & 2.40 & 0.37 \\
& Red & $2.63 \pm 0.35$ & 3.13 & 0.50 \\
& Green & $1.67 \pm 0.29$ & 1.88 & 0.21 \\
\hline \multirow{5}{*}{ Philips Luxeon Rebel } & Polar White & $1.40 \pm 0.03$ & 1.38 & -0.02 \\
& Warm White & $1.08 \pm 0.03$ & 1.04 & -0.04 \\
& Neutral White & $1.25 \pm 0.04$ & 1.20 & -0.05 \\
& Blue & $1.20 \pm 0.09$ & 1.08 & -0.12 \\
& Red & $1.04 \pm 0.07$ & 0.97 & -0.07 \\
& Green & $1.28 \pm 0.09$ & 1.15 & -0.13 \\
\hline \multirow{5}{*}{ Osram Golden Dragon } & White & $0.60 \pm 0.19$ & - & - \\
& Warm White & $0.70 \pm 0.17$ & 0.93 & 0.23 \\
& Blue & $0.63 \pm 0.12$ & - & - \\
& Red & $0.56 \pm 0.12$ & - & - \\
& Green & $0.70 \pm 0.13$ & - & - \\
\hline
\end{tabular}




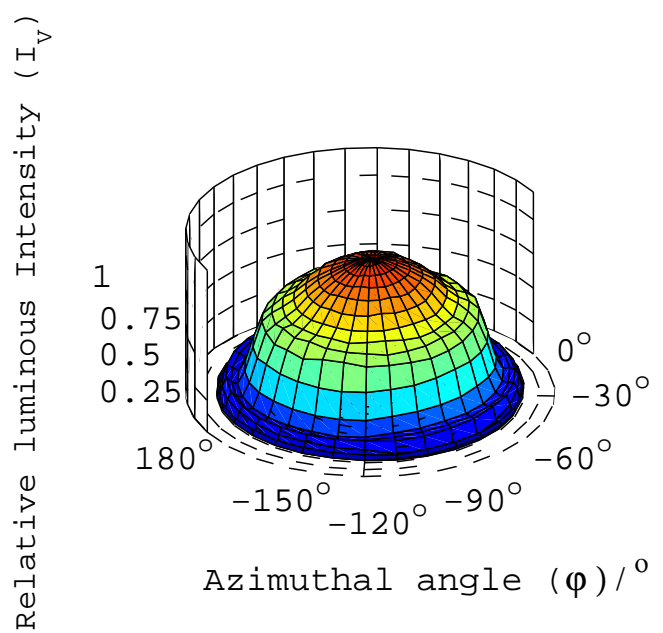

(a) Cree Xlamp LED

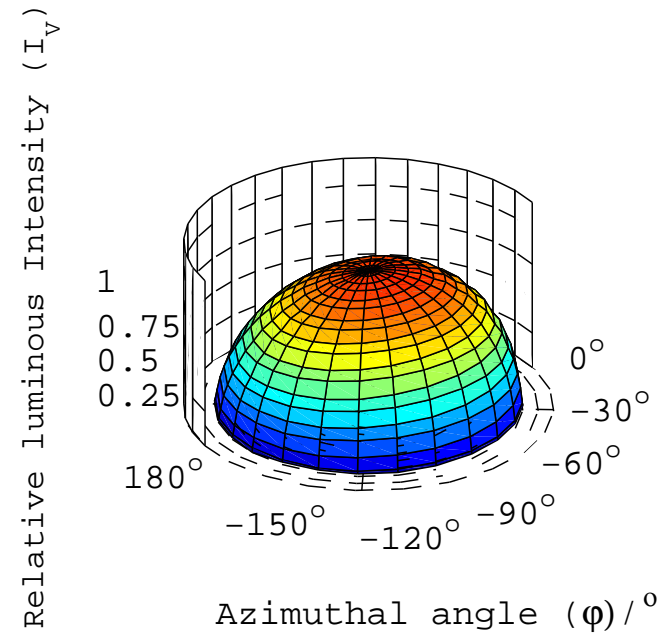

(c) Philips Luxeon Rebel LED

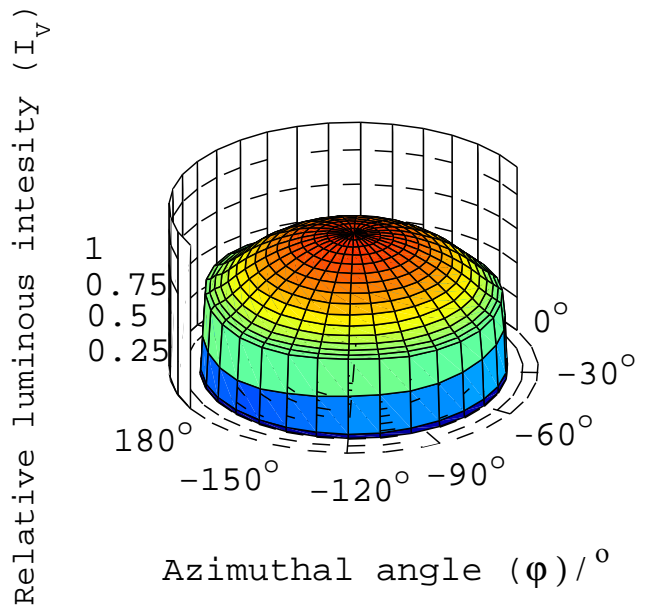

(e) Osram Golden Dragon LED

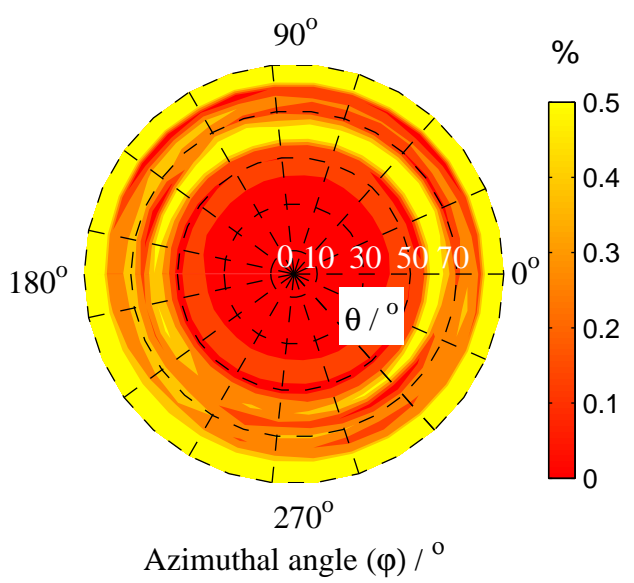

(b) Fitting deviation for Cree Xlamp LEDs

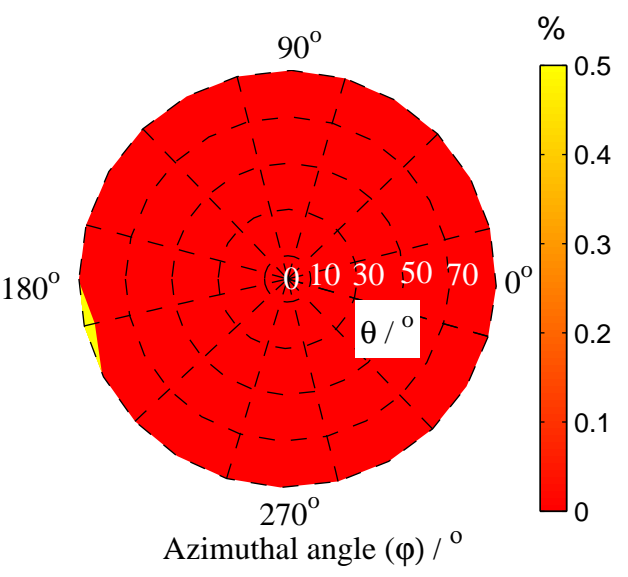

(d) Fitting deviation for Philips Luxeon Rebel LEDs

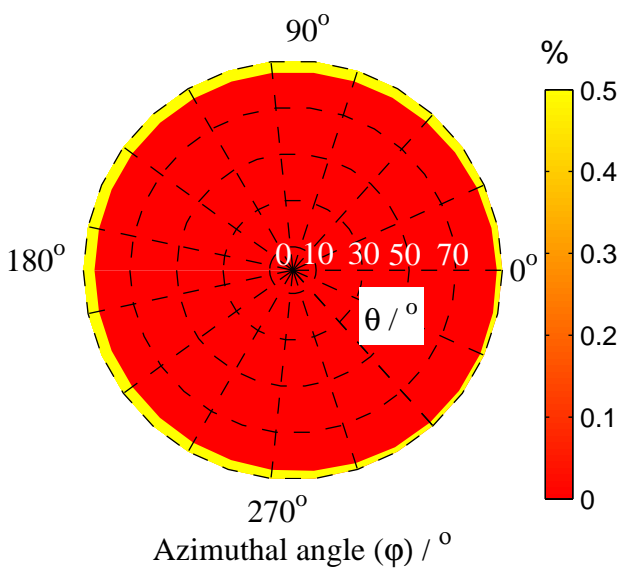

(f) Fitting deviation for Osram Golden Dragon LEDs

Figure 1. Luminous intensity distribution of three LEDs measured in the laboratory (a, c, e) and relative deviation between Zernike fitting and experimental data (b, d, f). 


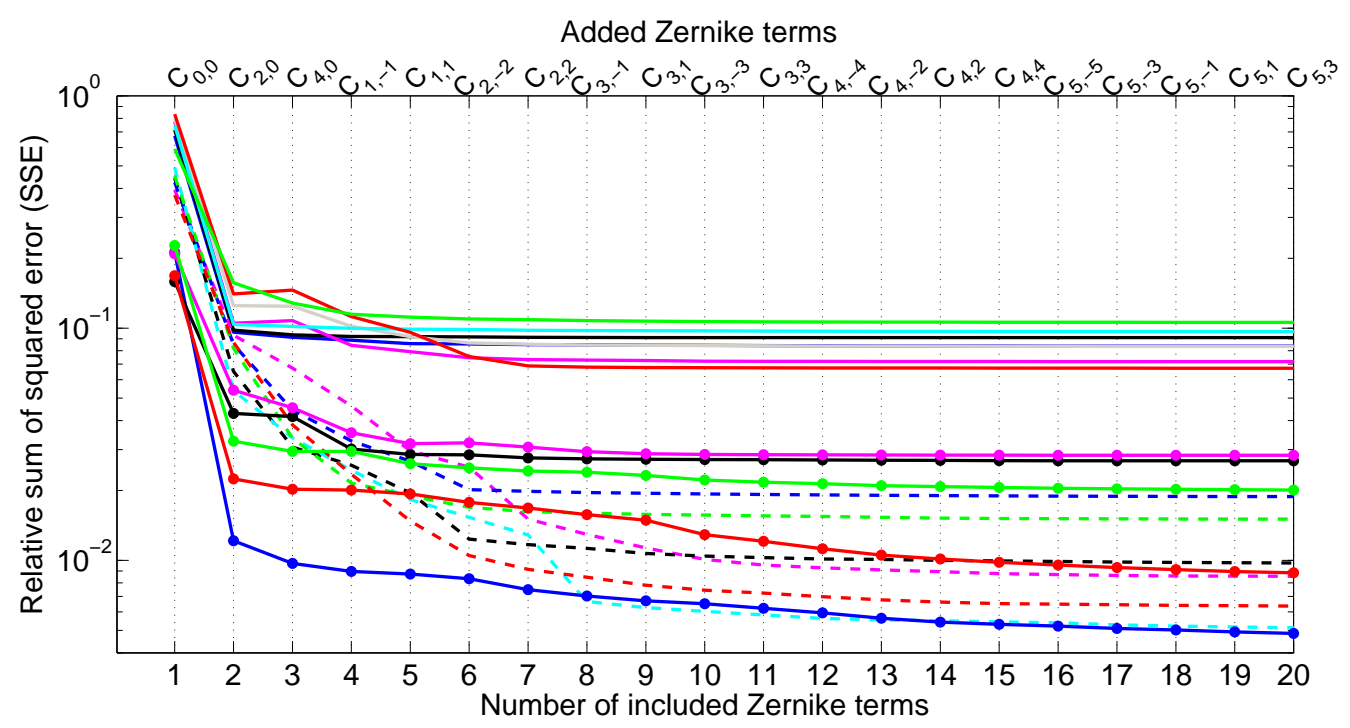

\begin{tabular}{l}
\hline - Blue Cree \\
- White Cree \\
- Warm white Cree \\
- Neutral white Cree \\
- Polar white Cree \\
- Red Cree \\
- Green Cree \\
-- - Warm white Luxeon \\
-- - White Luxeon \\
-- - Blue Luxeon \\
-- - Polar white Luxeon \\
--- Green Luxeon \\
-- - Red Luxeon \\
$\rightarrow-$ Blue Osram \\
$\rightarrow-$ White Osram \\
$\rightarrow-$ Warm white Osram \\
$\rightarrow-$ Red Osram \\
$\rightarrow$ Green Osram
\end{tabular}

Figure 2. Goodness of the fitting obtained by the Zernike approach. The relative sum of squared errors (SSE) (in semi-logarithm scale) is given for the 18 studied LEDs as a function of the number of Zernike terms used in the fitting.

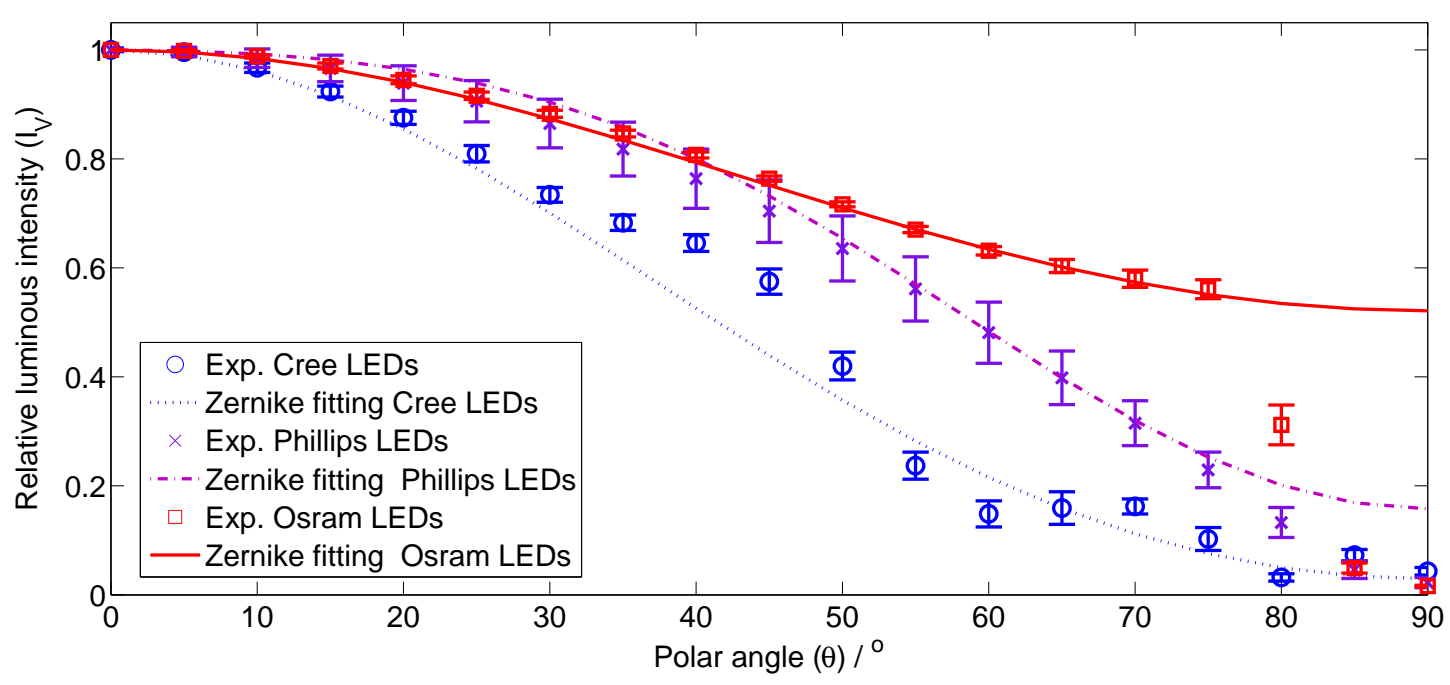

Figure 3. Relative luminous intensity versus polar angle. Experimental values as well as fitted values using Zernike polynomials are shown for three LEDs from different manufacturers 


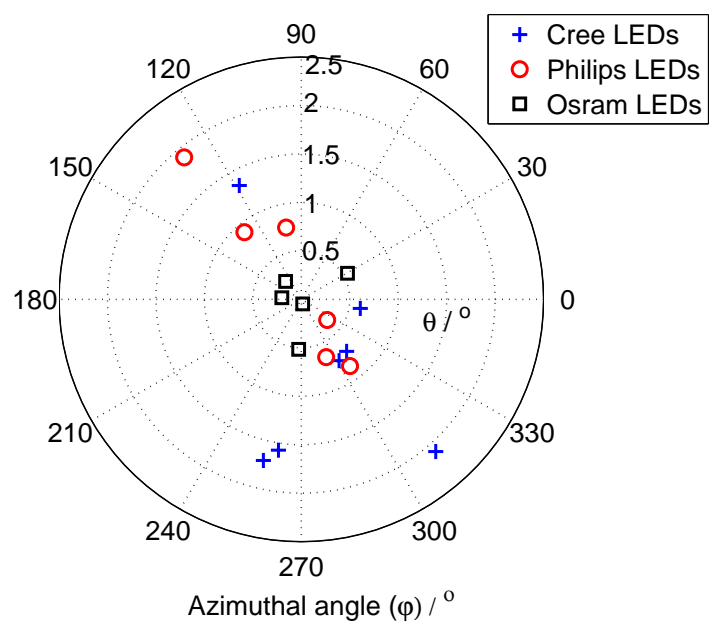

Figure 4. Direction of optical axis for one representative LED of each manufacturer.

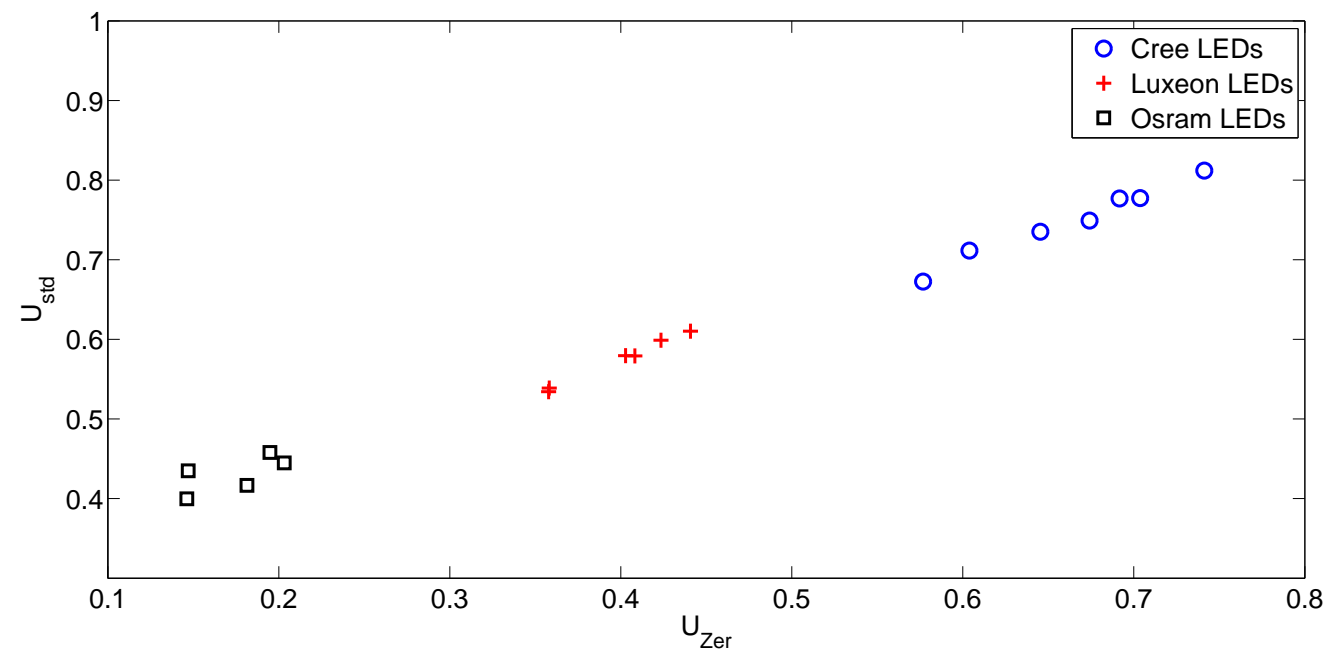

Figure 5. Relation between the inhomogeneity values calculated for one representative LED of each manufacturer. $U_{\text {std }}$ is the value calculated from the luminous intensity distribution while $U_{\text {Zer }}$ is the one calculated from the Zernike fit. 


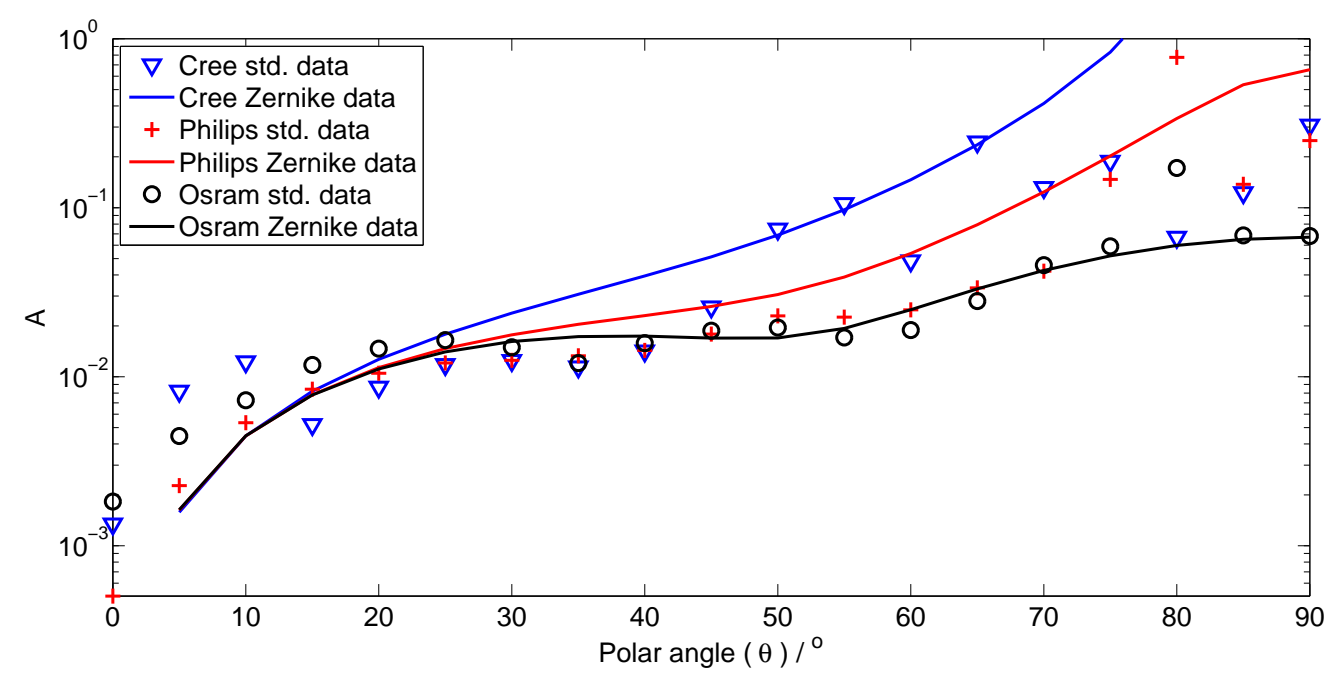

Figure 6. Anisotropy values from experimental luminous intensity values and from Zernike-based values, for one representative LED of each manufacturer. 